\title{
La necesidad de reconocer a la medicina del quemado como una nueva e independiente especialidad
}

\author{
Dr. Fortunato Benaim \\ Profesor Consultor en Cirugía Plástica de la Universidad del Salvador. Presidente de la Fundación del Quemado \\ Dr. Fortunato Benaim. Vicedecano de la Facultad de Ciencias de la Salud de la Universidad de Ciencias Empresariales \\ y Sociales. Miembro de Número de la Academia Nacional de Medicina. Profesor Honorario de Cirugía de la U.B.A. \\ Buenos Aires, Argentina.
}

La frecuente observación, aún en nuestros días, de pacientes con graves quemaduras atendidos en lugares no apropiados, que no cuentan con los recursos humanos y físicos necesarios para afrontar el tratamiento que tal patología requiere, ha motivado la presentación de este escrito.

José María del Río, en su Relato Oficial al Congreso Argentino de Cirugía realizado en Buenos Aires el año 1945, al abordar el tema "Quemaduras" (tema oficial) que ocupó una sesión plenaria, recordaba la frase de Dupuytren quien en 1839 afirmaba que "el tratamiento de las quemaduras ha sido objeto, en todos los tiempos, de las tentativas más bizarras (extravagantes) del empirismo" (1).

Resulta casi incomprensible que 175 años después (18392014) esta misma frase siga teniendo vigencia al comprobar que todavía se siguen utilizando aplicaciones locales no recomendables, o se asiste a un paciente grave sin contar con los recursos que actualmente ofrece el progreso de la Medicina.

Debe reconocerse que a partir del Siglo XX, al recurrirse a los injertos de piel autóloga para la reparación de las superficies cruentas originadas al extirparse quirúrgicamente el tejido necrótico generado por las quemaduras profundas (destrucción de todo el espesor de la piel), los cirujanos plásticos que se hicieron cargo de tal tratamiento aportaron un recurso terapéutico de indiscutible valor.

Publicaciones tradicionales aparecidas en las primeras décadas del Siglo XX, como el tratado Treatment of Burns de Harkins (1942) (2), el libro Skin Grafting in Burns de J. A. Barret Brown y F. McDowell (1943) (3) y muchos otros libros y artículos comunicando la experiencia obtenida por los cirujanos plásticos que se ocuparon del tema, son demostrativas del avance que significó el incorporar este recurso al tratamiento local y quirúrgico de las quemaduras.

Muchas víctimas de la Segunda Guerra Mundial debieron ser tratadas por graves secuelas como consecuencia de lesiones sufridas durante los combates, y la obra en 2 tomos de los cirujanos Harold Gillies y Ralph Millard, The Principles and Art of Plastic Surgery (1957) (4) en su Volumen I, describe con minuciosidad las ingeniosas técnicas de sus autores utilizando colgajos tubulares para el tratamiento de complicadas secuelas faciales y de otras localizaciones que afectaron a pilotos de la Fuerza Aérea Británica y a otros militares que participaron en esa conflagración.

Por mi parte, aporté la experiencia lograda a partir de 1948 al organizar en el Servicio de Cirugía del Hospital C. Argerich, cuya jefatura ejercía el Prof. A. Yódice, una unidad para asistir a pacientes quemados, presentando en el año $1952 \mathrm{mi}$ Tesis de Doctorado sobre el tema "Fisiopatología y Tratamiento de las Quemaduras. Resultados obtenidos con el Injerto de piel" (5), completando el texto con la documentación de los casos asistidos en ese período (1948-1952).

En los Congresos Latino-Americanos de Cirugía Plástica iniciados en la década de los 40 en Brasil (San Pablo y Río de Janeiro) y continuados luego cada 2 años en Argentina (Buenos Aires y Mar del Plata), Chile (Santiago y Viña del Mar), Uruguay (Montevideo y Punta del Este) y mantenidos hasta el presente con igual frecuencia, han sido presentadas numerosas contribuciones de cirujanos plásticos exponiendo su experiencia en el uso de injertos y colgajos para la corrección de variadas secuelas.

El empleo sistemático de los injertos cutáneos en el tratamiento de las quemaduras profundas, fue determinante para que en la práctica médica se apelara a los cirujanos plásticos para hacerse cargo de la asistencia de estos pacientes.

El año 1953 se crea en la ciudad de Buenos Aires el Instituto de Quemados, Cirugía Plástica y Reparadora, dependiente del Ministerio de Salud de la Nación; es decir un hospital completo, con personal médico (cirujanos, clínicos, especialistas, enfermeras, quinesiólogos, terapistas ocupacionales, asistentes sociales, nutricionistas, servicios centrales de Laboratorio, Radiología, Hemoterapia y Anatomía Patológica, dedicado al tratamiento de estos pacientes. Sin embargo, su nombre asociaba la Cirugía Plástica al tratamiento de las quemaduras ratificando el erróneo concepto de aceptar que esta compleja patología debe ser asistida por esta rama de la Cirugía.

En el $1^{\text {er }}$ Congreso Internacional de Investigaciones en Quemaduras realizado en la ciudad de Bethesda (Maryland, EE.UU.) el año 1960 (6), puede comprobarse al revisar su programa científico, que de los 57 temas tratados, sólo 10 fueron dedicados al tratamiento local y quirúrgico (es decir los que están a cargo de cirujanos), 5 a la investigación de homotrasplantes y 42 trabajos abordaron las alteraciones generales que caracterizan la evolución de las quemaduras graves y dan origen a complicaciones complejas que ponen en riesgo la vida del paciente, de los cuales 9 se refirieron a problemas generales, y los restantes: 9 a fluido terapia; 8 a cambios metabólicos y endocrinos; 3 a cambios hepáticos; 7 a infección; y 6 a toxinas y suero de convalecientes.

Este recuerdo destaca la importancia del aspecto clínico en el estudio y tratamiento de las quemaduras graves y pone en evidencia la necesidad de recurrir a los conocimientos inherentes a la Medicina Interna para la interpretación de las mencionadas alteraciones, y en consecuencia, aplicar las terapéuticas adecuadas para encarar cada situación.

Por tal motivo, en los servicios donde se internan estos pacientes para su tratamiento, se convoca a médicos clínicos (actualmente se recurre a los especialistas en terapia intensiva) para hacerse cargo del tratamiento general, ocupándose el cirujano plástico de las curaciones locales y de la reposición cutánea.

El año 1964, al realizarse en la ciudad de Caracas, Venezuela, el $2^{\circ}$ Congreso Latino-Americano de Cirugía Plástica, fui invitado a disertar sobre el tema "El paciente quemado como problema médico y social" (7).

En esa oportunidad, destaqué la necesidad de contar con un "grupo interdisciplinario de profesionales idóneos para el tratamiento integral" de los pacientes con quemaduras graves, pues además de los aspectos clínico-quirúrgicos, se requieren cuidados especiales de enfermeras y la participación de quinesiólogos, nutricionistas y apoyo psicológico, sin dejar de lado la intervención de otros profesionales y de los trabajadores o asistentes sociales, para contribuir a su reinserción social. 
Este concepto de tratamiento integral realizado por un grupo interdisciplinario, es hoy admitido sin reparos y recomendado como el medio más idóneo para asistir a pacientes con graves quemaduras (8-16).

El manejo de un grupo exige la presencia de un coordinador que sea el responsable de armonizar la acción de cada uno de sus integrantes. Suele ocurrir que el cirujano indica la necesidad de una intervención quirúrgica, pero el clínico considera que el paciente no está en condiciones de ser operado, y se plantea la necesidad de tomar una decisión.

En esas circunstancias, es indispensable que alguien asuma esa responsabilidad y determine cuál debe ser la actitud que debe adoptar el equipo para continuar con el tratamiento.

Esta situación es la que obliga a disponer de un especialista que con criterio clínico-quirúrgico y equilibrando ambos requerimientos, tenga la capacidad y los conocimientos necesarios para dirigir el grupo y tomar la decisión.

En el $13^{\circ}$ Congreso Internacional de Quemaduras organizado por la ISBI (International Society for Burn Injuries) en la ciudad de Montreal, Canadá, el año 2008, presenté un poster con el siguiente título Burn Medicine: should be an independent medical speciality? (Medicina del Quemado: ¿debiera ser una especialidad médica independiente?) sometiendo el interrogante a la opinión de los asistentes, a los que se invitaba a dejar su respuesta positiva o negativa con su tarjeta de identificación, para lo cual se agregó un dispositivo especial de recolección. Se recibieron 52 respuestas aportadas por congresistas de distintas latitudes, todas afirmativas, es decir, expresando su acuerdo en reconocer a la Medicina del Quemado como una nueva especialidad.

Quedó así demostrado que sería necesario encarar la formación de nuevos especialistas en Medicina del Quemado, y al no existir en nuestro medio programas completos de capacitación integral, para preparar esos nuevos especialistas decidimos desde el año 2006 iniciar el dictado de un Curso de Postgrado con el nombre de Diplomatura sobre la Fisiopatología y Tratamiento Integral de las Quemaduras (15).

Contamos con el respaldo de la UCES (Universidad de Ciencias Empresariales y Sociales) y se acordó que tuviera 2 años de duración, incluyendo en su programa de estudios recuerdos básicos de Fisiología y Fisiopatología, temas clínicos, quirúrgicos y de rehabilitación física y psicosocial, completándose la enseñanza con seminarios sobre: estadística, prevención, conducta en casos de catástrofe, categorización, planificación y organización de centros y normas para su acreditación, historia clínica especial e historia del conocimiento y tratamiento de las quemaduras. Como requisito para la inscripción, se exige haber completado una residencia en alguna de las siguientes especialidades: Cirugía General, Cirugía Plástica, Traumatología o Terapia Intensiva.

Esta Diplomatura será transformada en Carrera de Postgrado, y es la que brinda a los profesionales que han cursado las residencias mencionadas la capacidad agregada que exige el Ministerio para obtener la certificación respectiva.

Actualmente, estamos proponiendo la creación de una Residencia en Medicina del Quemado de 4 años de duración a la que podrán optar los médicos recién ingresados. Los 2 primeros años serán destinados a brindarles los conocimientos y prácticas de Medicina y Cirugía General, y los 2 últimos $\left(3^{\circ}\right.$ y $\left.4^{\circ}\right)$ se cursarán en un centro especializado de alta complejidad para que el residente tanga la oportunidad de familiarizarse con el manejo de estos pacientes, tanto en su tratamiento clínico general como en las intervenciones quirúrgicas que requieran.

Por otra parte, se ha solicitado al Ministerio de Salud de la Nación, en nota del 6 de Septiembre del año 2007, el reconocimiento de la Medicina del Quemado como especialidad independiente (15). En la actualidad se ha reiterado esta petición, respondiendo a los requisitos que el Ministerio exige, contando en esta oportunidad con el aval de la Academia Nacional de Medicina y la Asociación Argentina de Quemaduras.

En los antecedentes que acompañaron a la nota y como argumentos que respaldan la solicitud, se aportan datos sobre la existencia actual en varios hospitales de Argentina y en otros países, de unidades y servicios para el tratamiento de las quemaduras (aunque aún muchos de ellos no cuentan con todos los recursos que hoy ofrecen la tecnología y el progreso de la Medicina), y también la creación de Sociedades Científicas Nacionales e Internacionales que agrupan a los profesionales interesados en el tema; la publicación de revistas científicas especializadas y la realización permanente y periódica de congresos nacionales, regionales e internacionales.

La falta de ese reconocimiento oficial permite que en la actualidad, el tratamiento de estos pacientes pueda efectuarse en servicios que no cuentan con los elementos apropiados, obteniéndose como consecuencia resultados insatisfactorios con morbimortalidad mayor de la que puede lograrse con la aplicación racional de los recursos que el progreso de la Medicina hoy nos ofrece.

Como reflexión final, cabe señalar que es de esperar que si se logra el reconocimiento de la Medicina del Quemado como especialidad básica, el tratamiento de estos pacientes deberá realizarse en el futuro exclusivamente en servicios preparados para efectuarlo, que cuenten con personal idóneo certificado por las sociedades científicas y planta física y equipamiento adecuados para brindar a estos pacientes la asistencia de calidad que merecen y que los avances científicos aportan.

La habilitación y acreditación de nuevos servicios categorizados adecuadamente y la capacitación completa de los profesionales ratificada por la certificación que otorguen las sociedades científicas respectivas, serán la mejor garantía para el correcto tratamiento de esta complicada patología.

De esta manera, la frase de Dupuytren mencionada al comienzo de esta nota quedaría en la historia de la especialidad como un extravagante recuerdo.

\section{Bibliografía}

1. Del Río J.M.: Quemaduras. Ed. López. Bs. As., Argentina, 1947.

2. Harkins H.: The treatment of burns. Ch. Thomas. Springfield, 1942.

3. Brown J.B.; McDowell, F.: Sking grafting of burns. Ed. J.B. Lippincott Company. Philadelphia, 1943.

4. Gillies H.; Millard R.: The principles and art of plastic surgery. Little, Brown and Company. Boston - Toronto, 1957.

5. Benaim F.: Fisiopatología y tratamiento de las quemaduras. Resultados obtenidos con el injerto de piel. Tesis de Doctorado. Fac. de Medicina, U.B.A., 1952.

6. Artz C.P.: Research in Burns. The proceedings of the First International Congress on Research in Burns at the National Naval Medical Center. Bethesda, Mariland, 1960. Co-published by American Institute of Biological Sciences. Washington D.C. and F. A. Davis Company, Philadelphia.

7. Benaim F.: El quemado como problema médico-social. Notas del ayer (1967). Rev. Arg. Quem. 1991, Vol. 14: 1.

8. Artz C.P.; Moncrief J.A.; Pruitt B.A.: Burns a Team Approach. W.B. Saunders, Philadelphia, 1979.

9. Gonzalez Ulloa M.; Stevens E.: Quemaduras Humanas. Ed. Interamericana S.A. México, 1960.

10. Russo A d C.: Tratamento das queimaduras. Ed. Sarvier. Sao Paulo. 2* Edición. 1976.

11. Bracho J.B.; Bracho J.J.; Bracho J.E.: Quemaduras. Ed. Imprenta Teran. Quito, Ecuador. 1994.

12. Garcés M.; Artigas N R.: Quemaduras. Ed. Antonio Yuri P. Sgo. Chile, 1995

13. Bendlin A.; Linares H.; Benaim F.: Tratado de Quemaduras. Edit. Interamericana Mc Graw Hill. México, 1997.

14. Maciel E.; Serra MC.: Tratado de Queimaduras. Ed. Atheneu. Sao Paulo, Río de Janeiro. Ribeirao Preto. Belo Horizonte, Brazil, 2004.

15. Benaim F.: 60 Años dedicados a la Medicina del Quemado. Recuerdos autobiográficos y aportes científicos. Ed. Fundación OSDE, 2010.

16. Bolgiani A.; Maciel E.M.; Serra M.C.: Quemaduras. Conductas clínicas y quirúrgicas. Ed. Atheneu. Sao Paulo, Río de Janeiro, Belo Horizonte, Brazil, 2013. 